\title{
Candidate main-field models for producing the 9th generation IGRF
}

\author{
Mioara Mandea* \\ Institut de Physique du Globe de Paris, 4 place Jussieu, 75252 Paris Cedex 05, France
}

(Received January 11, 2005; Revised May 3, 2005; Accepted May 3, 2005)

\begin{abstract}
This paper presents the various candidate models used in deriving the 9th generation IGRF. Based on notes submitted to the IAGA working group V-MOD with the Gauss coefficients, a brief description of the data used and the method of modelling for each of the candidate models is given. The six candidate models for epoch 1995.0 and the five for epoch 2000.0 are presented. Improvements gained by the new models are also discussed.

Key words: IGRF, DGRF, modeling, core field, secular variation.
\end{abstract}

\section{Introduction}

The largely non-specialist community of users favours an established, regular routine for updates of the IGRF models and for designation of the DGRF models. One of the central issues is to collect candidate models for the new IGRF or DGRF, evaluate the candidate models, and adopt the final IGRF or/and DGRF models. Generally, this occurs every 5 years, and is one of the main tasks of the IAGA working group, for time being named V-MOD, formerly called V-8 Analysis of the Global and Regional Field and its Secular Variation. The large community using these models have already stated clearly that it is necessary to preserve simplicity and ease of identification of the standard. Therefore, the working group V-MOD maintains the traditional 5-year recipe, except for the 9th generation IGRF which was revised after only 3 years (for more details see the web page $\left.^{1}\right)$, with a spherical harmonic expansion for the mainfield model up to degree/order $N_{i}=10$ (for models prior to 2000), or $N_{i}=13$ (for models starting in 2000), and with an expansion for the predicted secular variation $N_{S V}=8$.

In this paper a summary, in a standard form, of the various candidate models for the 9th generation IGRF is given. Based on notes submitted to V-MOD with the Gauss coefficients, a brief description of the data used and method of modelling for each of the candidate models is given (for the most complete description of candidate models see the web page ${ }^{2}$. The aim of this work is to preserve information about the candidate models considered in producing the 9th generation IGRF, as only a few of them are presented in this issue. Each candidate model is identified by the name attributed during the evaluation processes. Table 1 summarizes the candidate models for the 9th generation IGRF.

\footnotetext{
*Now at GeoForschungsZentrum Potsdam.
}

Copyright (c) The Society of Geomagnetism and Earth, Planetary and Space Sciences (SGEPSS); The Seismological Society of Japan; The Volcanological Society of Japan; The Geodetic Society of Japan; The Japanese Society for Planetary Sciences; TERRAPUB.

\section{Candidate Models for the Epoch 1995.0 2.1 DGRF1995-BGS}

This model was proposed by A. Thomson and S. Macmillan.

Data The dataset used covers the 1991-1999 time span and it contains near-Earth and total field satellite data provided by POGS satellite.

Annual mean $X, Y, Z$ values for 1991-1998 from observatories were selected. Crustal biases were computed where possible, by comparison of the 1999 annual means with an Ørsted-only based model. For 150 observatories these crustal biases were deducted and removed from the annual means; for 55 no bias corrections have been applied.

Repeat stations occupied between 1991.0-1999.0 were also used. After removing outliers and observations with large crustal fields, based on comparison with 8th generation IGRF and eliminating stations with $|Z, F|$ residuals $\geq 500 \mathrm{nT}$ and $|X, Y|$ residuals $\geq 500 \sqrt{2} \mathrm{nT}, 1320$ repeat stations were kept. Some 90 one-off surveys were added, after processing them in the same way.

From 1993.05 to 1994.23 one-minute mean values of Project MAGNET aeromagnetic vector observations were considered. After selection over periods when the geomagnetic planetary three-hour-range index $\mathrm{Kp}$ is $\mathrm{Kp}<2+$ and after applying the same rejecting criteria as for the repeat stations, 22640 data were used.

Marine total intensity data covered 1991.0-1999.0 period. The final 4473 marine data were obtained after selecting every second point along survey tracks; $110 \mathrm{~km}$ alongtrack means were computed from a minimum 25 data with $\mathrm{Kp} \leq 2+$ and $|F|$ residuals $\leq 500 \mathrm{nT}$ when comparing with the 8th generation IGRF.

POGS satellite scalar data, between 1991.03-1993.58, were selected according to the following criteria: $\mathrm{Kp} \leq 1+$, $-15 \leq D_{s t} \leq+5$ (where $D_{s t}$ is a geomagnetic index which monitors the world wide magnetic storm level), 22:00 $\leq$ local time $\leq$ 05:00, solar zenith angle filtered (data rejected where ionosphere below satellite is sunlit), and an outlier rejection for $|F|$ residuals $\geq 100 \mathrm{nT}$ when comparing with

\footnotetext{
${ }^{1}$ http://www.iugg.org/IAGA/iaga_pages/science/division_5.htm.

${ }^{2}$ http://www.ipgp.jussieu.fr/ mioara/dgrf/dgrf_page.html.
} 
Table 1. Candidate models for the 9th generation IGRF.

\begin{tabular}{ll}
\hline Model-Abbreviation & Authors (countries) \\
\hline DGRF1995-BGS & Thomson and Macmillan (UK) \\
DGRF1995-CM & Olsen, Lowes, Sabaka (Denmark, UK, USA) \\
DGRF1995-GFZ & Maus, Lühr, Rother, Mai, Mandea (Germany, France) \\
DGRF1995-GFZ2 & Wardinski and Holme (Germany, UK) \\
DGRF1995-IPGP & Chambodut, Langlais, Mandea (France, USA) \\
DGRF1995-IZM & Bondar and Golovkov (Russia) \\
DGRF2000-BGS & Lesur, Thomson, Macmillan (UK) \\
DGRF2000-CM & Olsen, Lowes, Sabaka (Denmark, UK, USA) \\
DGRF2000-OSVM & Olsen, Lowes, Sabaka (Denmark, UK, USA) \\
DGRF2000-GFZ & Maus, Lühr, Rother, Mai (Germany) \\
DGRF2000-IPGP & Chambodut, Langlais, Mandea (France, USA) \\
\hline
\end{tabular}

the 8th generation IGRF. Both the near-surface and satellite datasets were further decimated by selecting separately a maximum 7 data locations per equal-area tessera of which there were 1654 covering the Earth's surface. The selection used the magnetically quietest data and for the near-surface data used those from observatories, repeat stations, oneoff surveys, Project MAGNET and marine surveys in that order.

Modelling method Data were weighted in the model solution. The relative weights for both satellite and surface data, in terms of data quality and crustal field signature, were determined by iteratively re-weighting the input data according to fits to interim models, derived at each step of a six stage iteration process. Equal-area weighting was also applied to all data. All data were reduced to the epoch 1995.0 prior to main-field model derivation, using annual secular-variation models prepared by BGS from observatory and other data.

Three models were computed and compared with the following parameterizations (based on the input data sets given in parentheses): (A) $N_{i}=13$ internal, $N_{e}=1$ external (POGS + surface data). No $D_{s t}$ or seasonal, i.e. time, dependence was provided for the external terms. (B) $N_{i}=13$ internal (POGS + surface data). (C) $N_{i}=10$ internal (POGS + surface data; and surface data only). The BGS candidate main-field model for 1995.0 is the truncation to $N_{i}=10$ of model (A). For this model formal standard deviations were given.

\subsection{DGRF1995-CM}

This model was proposed by $N$. Olsen, F. Lowes and T. Sabaka, and is described in more detail in Olsen et al. (2005).

The basis of this candidate model is a $N_{i} \leq 14$ truncation of the internal field terms of the Comprehensive Model CM3e_J-2. This is an extended version (Sabaka et al., 2004), of the model described in Sabaka et al. (2002). For more details see also Olsen et al. (2005).

Data The original model had been derived from quiettime MAGSAT and POGO satellite and observatory hourly mean measurements for the period 1960-1985. Scalar data from CHAMP and vector and scalar data from Ørsted have been incorporated, in extension, along with all available observatory data till 2000 .

Modelling method A model, designated CM3 (Comprehensive Model: phase 3), the third in a series of efforts to co-estimate fields from various sources is the basis of the candidate model. The CM3 model also accounts for main field influences on the magnetosphere, main field and solar activity influences on the ionosphere, seasonal influences on the coupling currents, a priori characterization of the influence of the ionosphere and the magnetosphere on Earth-induced fields, and an explicit parameterization and estimation of the lithospheric field. The result is a model that describes well the 591432 data with 16594 parameters, implying a data-to-parameter ratio of 36 , which is larger than several popular field models. The candidate main-field model for 1995.0 is the truncation to $N_{i}=10$ of the model $\mathrm{CM} 3 \mathrm{e} \_\mathrm{J}-2$.

\subsection{DGRF1995-GFZ}

This model was proposed by S. Maus, H. Lühr, M. Rother, W. Mai and M. Mandea.

Data The dataset used contains satellite vector data provided by the Ørsted satellite (21-Apr-1999 to 30-Sep2002) and by the CHAMP satellite (15-May-2001 to 30Sep-2002). Initial datasets were then selected according to the following criteria: $\mathrm{Kp} \leq 2,-20 \leq D_{s t} \leq+20$, local time 00:00 to 06:00 (except for early Ørsted data, when due to scarce data over the Atlantic, the local time is 22:00 to 06:00). Finally, a selection of quiet tracks by an algorithm which chooses a dense spatial coverage, by taking into account the local track-RMS against a crude initial model was applied. This selection was undertaken separately for polar and mid-latitude track segments.

Observatory annual means are also used from 1994 to 2001. On visual inspection, data provided by the following observatories were rejected: AAE, ASC, ASH, CSY, CWE, HVN, KOD, KRC, MLT, MNK, PND, VSK (indicated by their IAGA codes).

Modelling method Satellite data were weighted to give roughly uniform coverage over the sphere for each dataset. The Ørsted dataset was divided in two slots to achieve an equal temporal coverage. Observatory data were 
equally weighted. The three datasets were combined with the same weight for the satellite datasets (40\% each) and with a smaller weight $(20 \%)$ for the observatory data.

In the least squares inversion the solved internal contributions are the main field, the secular variation and the secular acceleration (for the acceleration the degrees 8-10 were damped to impose a decreasing spectrum). Satellite data were corrected for $D_{s t}$ assuming $q_{0}^{1}=0.72 D_{s t}$ and $g_{1}^{0}=0.3 q_{0}^{1}$. The internal field expansion is $N_{i}=10$. For the quadratic secular variation the expansion degree is $N_{S V}=10$, by using combined satellite and observatory data. An estimation of standard deviation was obtained as difference between extrapolations using only odd-year, or even-year, observatory data.

\subsection{DGRF1995-GFZ2}

This model was proposed by I. Wardinski and R. Holme.

Data The observatory annual means (the observatory number varying between 138 for 2000 up to 184 for 1986) and a few repeat stations (varying between 0 for 1980 and 52 for 1990), were used to estimate the continuous secular variation from 1980 to 2000 . To minimize the misfit to the data two models were used as end constraints: a MAGSAT satellite model (Cain et al., 1967), for the beginning of the interval and one derived from Ørsted data (OSVM model, see Olsen, 2002), at the end of the interval.

Modelling method A cubic B-spline secular-variation basis is used to compute a time-dependent model between MAGSAT (1980) and Ørsted epochs (2000). The modelling method consisted of damped least squares to $N_{i}=10$, with cubic B-splines for time variation (knot spacing 2 years), with a temporal smoothing based on the second time-derivative, and a spatial norm based on the radial field, both at the core-mantle boundary.

An iterative weighted least square approach was applied, at first all data being equally treated. From this new weights were obtained for each observatory, which were used in the second model. In the second model the data were reweighted. In the third iteration step data outside the 2standard deviation interval from the second model were discarded. The final model was obtained within 1-standard deviation. This proposed candidate model for 1995.0 epoch was extracted from the 20-year series of models.

\subsection{DGRF1995-IPGP}

This model was proposed by A. Chambodut, B. Langlais and M. Mandea, and is decribed in more detail in Chambodut et al. (2005).

Data Annual mean values covering the 1994.5-2000.5 time span, for a fixed set of 112 observatories were used. When possible, annual means were considered back to 1979 , in order to check the time-series for consistency and the crustal biases. A jump was applied for TUC in 1996, and data for SYO were interpolated for 1997.5 and 2000.5. A main-field model for the epoch 2000.0, based only on Ørsted satellite data was also used.

Modelling method Because of the particularly uneven distribution of observatory data, they were weighted according to regional density, using the scheme described in Langlais and Mandea (2000). Undamped least squares inversion was applied for each year, so seven main-field models were obtained, up to degree $N_{i}=8$. A mean secular- variation model for 1995-2000 was computed from these main-field models. For degrees 9-10, the 2000.0 values of the Ørsted secular-variation model were kept. Applying this mixed secular-variation model to the candidate main-field model for epoch 2000.0, the 1995.0 model was obtained by an extrapolation back in time.

\subsection{DGRF1995-IZM}

This model was proposed by T. Bondar and V. Golovkov.

Data Observatory annual means for the period 19802000 were used indirectly, to produce a space-time model. For this model synthetic datasets derived from the 5th generation IGRF (essentially the core terms of a truncated MAGSAT model) and from Ørsted OSVM model (Olsen, 2002), were also considered.

Modelling method The main steps in obtaining the proposed candidate model were as following:

(a) To compute synthetic data, a preliminary model M1 for 1980-2000 was produced; this was quadratic in time. By imposing a 1980.0 main-field model (5th generation IGRF), and a 2000.0 main-field model (OSVM), and a linear secular-variation model (Olsen, 2002), the second derivatives of the coefficients were estimated.

(b) Observatory data, with gaps filled in from synthetic data from model M1, were analyzed to give a spacetime model M3, using the Natural Orthogonal Component (NOC) functions for the time variation.

(c) Step (a) was repeated, but this time for 1991-2000 period, using the 1991.0 value of M3, and the 2000.0 mainfield and its secular variation given by from OSVM model (Olsen, 2002), to produce the quadratic terms of a new model, called M4.

(d) The submitted model is the 1995.0 value of M4.

\section{DGRF2000 Candidate Models 3.1 DGRF2000-BGS}

This model was proposed by V. Lesur, A. Thomson and $S$. Macmillan.

Data This model is mainly based on Ørsted data, selected between the years 1999.25 and 2002.0. The selection criteria used were: $\mathrm{Kp} \leq 1+,-15 \leq D_{s t} / R C \leq+5$, 22:00 $\leq$ local time $\leq$ 05:00, for IMF (Interplanetary Magnetic Field) components: $-5 \mathrm{nT} \leq \mathrm{IMF} B_{x}$, IMF $B_{y} \leq 5$ $\mathrm{nT},-2 \mathrm{nT} \leq \mathrm{IMF} B_{z} \leq 5 \mathrm{nT}$, solar wind velocity $v \leq$ $450 \mathrm{~km} \mathrm{~s}^{-1}$ and the ionospheric E-region below satellite not sunlit.

Hourly mean vector data were selected from 119 observatories over the same period of time. The selection criteria for $\mathrm{Kp}, D_{s t}$ index and local time are the same. To reduce the amount of external and field-aligned current contributions at high latitude, observatory vector data outside $-40^{\circ}$ to $40^{\circ}$ geographic latitude were projected onto the direction of an a priori model.

Modelling method The standard deviations associated with the data are $2 \mathrm{nT}$ for satellite vector data and scalar data in the range $-55^{\circ}$ to $55^{\circ}$ geomagnetic latitude, $5 \mathrm{nT}$ for observatory vector data and remaining scalar satellite data and $10 \mathrm{nT}$ for observatory scalar data. The inverse of the covariance matrix was used to weight the data. A further weight for regional density ( $\sin ($ colat $)$ ) was applied to the satellite data only. 
An undamped iterative least-squares scheme, with iterative re-weighting of datasets was used. The internal terms are computed up to $N_{i}=20, N_{S V}=10$, external terms to $N_{e}=2$, with $D_{s t}$ variation of degree 1 (and induced) terms (for satellite data, only). Annual and semi-annual variations in time of the internal and external zonal coefficients were estimated up to degree 2. Crustal biases for observatory data were also estimated. The candidate model was extracted (truncated at $N_{i}=13$ ) from the above more comprehensive model.

\subsection{DGRF2000-CM}

This model was proposed by $N$. Olsen, F. Lowes and T. Sabaka, and is described in more detail in Olsen et al. (2005).

The basis of this candidate model is the $N_{i} \leq 14$ truncation of the internal field terms of the Comprehensive Model CM3e_J-2 (for more details see the DGRF1995-CM and the references therein). The proposed model is a truncation at $N_{i}=13$ of the 2000.0 static internal terms.

\subsection{DGRF2000-OSVM}

This model was proposed by $N$. Olsen, F. Lowes and T. Sabaka, and is described in more detail in Olsen et al. (2005).

The basis of this candidate model is the $N_{i} \leq 14$ truncation of the internal field terms of the OSVM model fully described in Olsen (2002), but with a correction made for the leakage of ionospheric field.

Data Ørsted satellite data are mainly used: vector data in the range $-50^{\circ}$ to $50^{\circ}$ geomagnetic latitude and Ørsted scalar data for geomagnetic latitudes greater than $50^{\circ}$ and in non-polar regions, to fill gaps. The data were selected using the following criteria: $\mathrm{Kp} \leq 1+$ and $\mathrm{Kp} \leq 2 o$ for previous 3 hours, $-10 \leq D_{s t} \leq+10,22: 00 \leq$ local time $\leq$ 05:00, $-3 \mathrm{nT} \leq \mathrm{IMF} B_{z} \leq 3 \mathrm{nT}$. These data were decimated to roughly equal area.

About 110 observatories were considered. The midnight values for quiet days, for the period 1998-2000 were used to estimate a secular-variation model.

Modelling method Anisotropic weights were applied to Ørsted vector data. The OSVM model was obtained by applying a partially damped iterative least-squares method, with iterative re-weighting of data-sets. The expansion was developed up to $N_{i}=29$ for the internal part, $N_{e}=2$ for the external part and a $D_{s t}$ for degree 1 terms. Annual and semi-annual variations in time of the internal and external zonal coefficients were estimated up to degree 2 .

The candidate model differs from the OSVM model by:

1) The OSVM had significant leakage of the average ionospheric field into the internal coefficients. In terms of mean-square vector field the leakage amounted to 39 $\mathrm{nT}^{2}$. To produce the candidate model this estimate was subtracted from the OSVM coefficients with degree smaller than 9 .

2) Leakage of the day-to-day variations of the ionospheric field meant that the quoted variances for the axial and near-axial coefficients were too small. Conversely, for other coefficients the quoted variances were too large. A smoothly varying correction factor was estimated, which has been applied to the original OSVM variance estimates.

3) The authors estimated (somewhat arbitrarily) that the correction applied in 1) is accurate to about $12 \%$ for each coefficient, corresponding to a (pseudo-random) variance equal to about $25 \%$ of the square of the coefficient. This variance contribution was added to the one estimated in 2).

4) A $195 \times 195$ covariance matrix is estimated (fairly arbitrarily) using the final variance estimates $(2+3)$, and the original OSVM correlation matrix, truncated to $N \leq 14$.

\subsection{DGRF2000-GFZ}

This model was proposed by S. Maus, H. Lühr, M. Rother, W. Mai.

The candidate model for 2000.0 was derived from an initial model estimated from a combined set of Ørsted and CHAMP satellite vector data.

Data Satellite Ørsted vector data from 21-Apr-1999 to 30-Sep-2002 and CHAMP vector data from 15-May-2001 to 30-Sep-2002 were used. For CHAMP, a static correction for the set of angles between the star camera reference system and the vector magnetometer was co-estimated in the inversion. The data were selected using the following criteria: $\mathrm{Kp} \leq 2,-20 \leq D_{s t} \leq+20,00: 00 \leq$ local time $\leq$ 06:00 or 22:00 $\leq$ local time $\leq$ 06:00 for early $\emptyset$ rsted due to scarce data over the Atlantic. The quiet tracks were selected by an algorithm which chooses a dense spatial coverage, taking into account the local track-RMS against a crude initial model.

Modelling method In the inversion, 50\% weight was given to Ørsted vector data and $50 \%$ weight to CHAMP vector data. The Ørsted data were subdivided into two subsets, each with $25 \%$ weight. The individual data were weighted to achieve equal data weight over the sphere within each data set. A partially damped least-squares scheme was used. The internal terms are computed up to $N_{i}=15, N_{S V}=15, N_{A C}=10$ (degrees 14-15 of secular variation $S V$ and 8-10 of secular acceleration $A C$ were damped to impose decreasing spectra). External terms are estimated up to $N_{e}=2$, assuming $q_{1}^{0}=0.72 D_{s t}$ and $g_{1}^{0}=0.3 q_{1}^{0}$ in geomagnetic coordinates. Since the magnetosphere is best described in Geocentric Solar Magnetospheric (GSM) coordinates, a 2nd degree external field in GSM was co-estimated in these coordinates. The coefficient $q_{1}^{0}$ in geomagnetic coordinates was co-estimated in the inversion to be $-16.62 \mathrm{nT}$.

The residuals between the final model (only up to degree 13, but including secular variation and secular acceleration) individual data were given. The Ørsted residuals are lower than the CHAMP residuals, because the Ørsted data were median filtered against an initial model to decrease the star camera noise.

\subsection{DGRF2000-IPGP}

This model was proposed by A. Chambodut, B. Langlais and M. Mandea, and is decribed in more detail in Chambodut et al. (2005).

Data This candidate main-field model was calculated from all available vector and scalar Ørsted satellite data, from April 1999 to September 2002. Vector data were used in the range $-50^{\circ}$ to $50^{\circ}$ geomagnetic latitude and scalar data for geomagnetic latitudes greater than $50^{\circ}$ and in nonpolar regions, when vector data were missing. The data were selected using the following criteria: $\mathrm{Kp} \leq 1+$ and $\mathrm{Kp} \leq 2-$ for previous 3 hours, $-5 \leq D_{s t} \leq+5$ and 

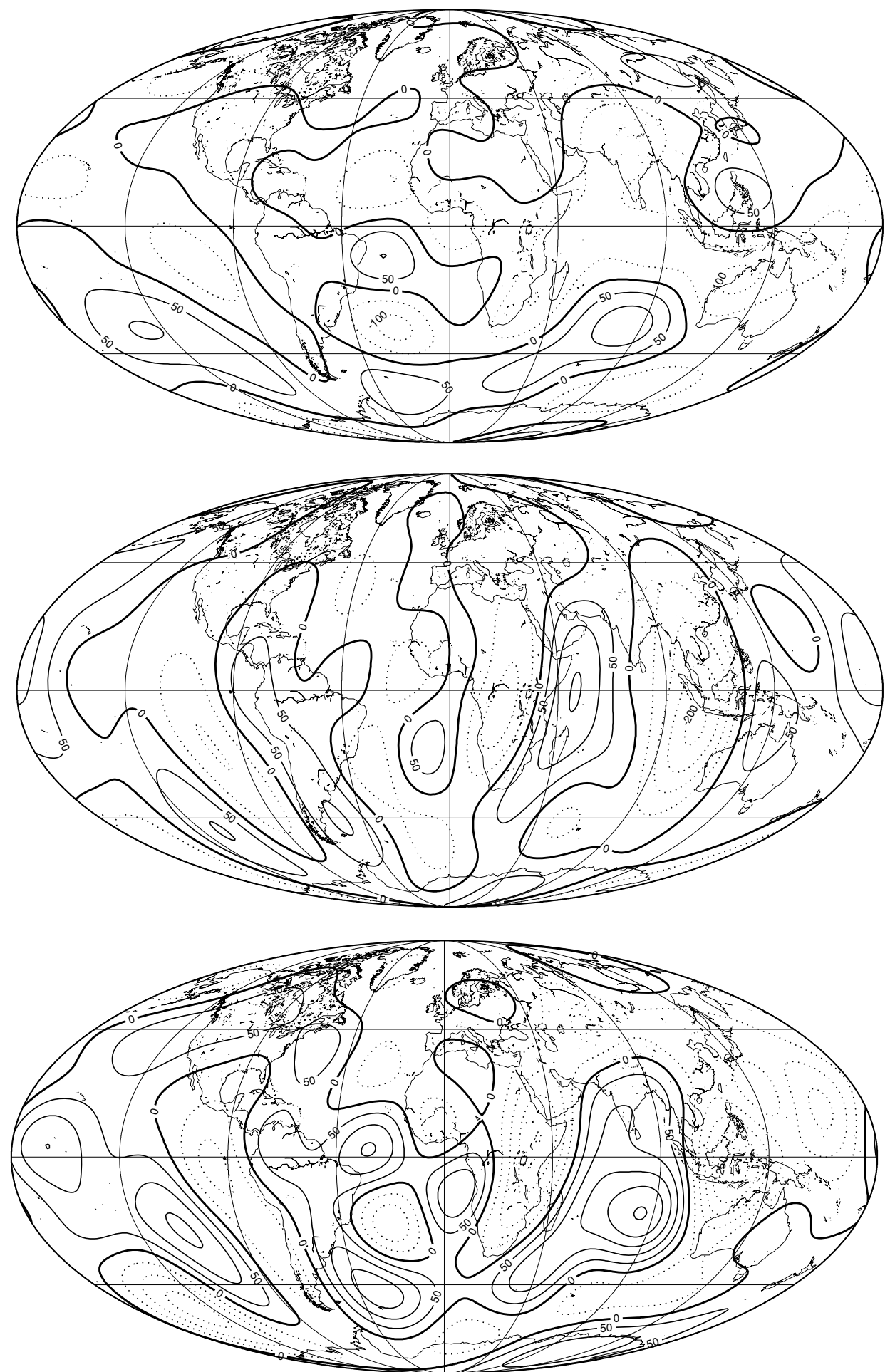

Fig. 1. Differences in $X, Y$ and $Z$ componenets from IGRF and DGRF models for epoch 1995.0. The countour interval is $50 \mathrm{nT}$.

$\left|d D_{s t}(t) / d t\right| \leq 3 \mathrm{nT} /$ hour and only measurements made in the real shadow side of the Earth (Chambodut et al., 2003).

Modelling method For the inversion process data were randomly selected (10 data per $3^{\circ} \times 3^{\circ}$ equiangular bin). A geographic weighting scheme was applied on satellite datasets and the anisotropy of star camera accuracy considered (Holme, 2000). A undamped iterative least squares routine was used, with the internal terms computed up to $N_{i}=29$ and $N_{S V}=10$, the external terms to $N_{e}=2$, with $D_{s t}$ dependence for degree 1 .
Using this first model all data were tested, and outlying data removed (for residual larger than $5 \mathrm{nT}$ ). Again, the remaining data were randomly selected, as in the initial model, and a new model computed with $N_{i}=20$ and $N_{S V}=8$ (imposed for degree/order 9 and 10), $N_{e}=2$, with $D_{s t}$ dependence for degree 1 . The proposed candidate model was this last model truncated to include only coefficients up to degree $N_{i}=13$. 

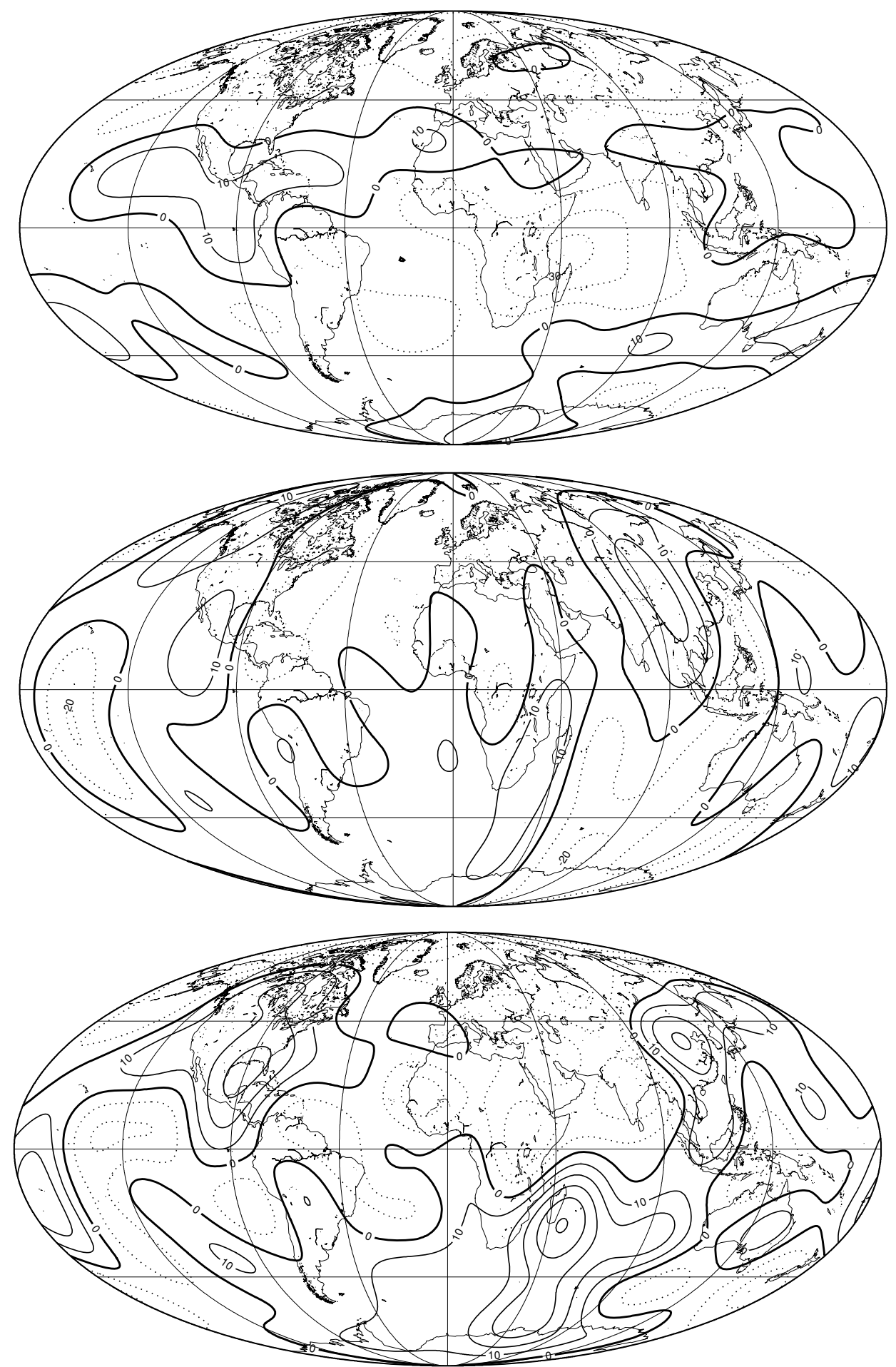

Fig. 2. Differences in $X, Y$ and $Z$ componenets from IGRF and DGRF models for epoch 2000.0. The contour interval is $10 \mathrm{nT}$. The DGRF2000 model was considered up to degree/order 10, as was the IGRF2000.

\section{Conclusions}

The aim of working group V-MOD of the IAGA Division $\mathrm{V}$ is to promote and coordinate international efforts to model and analyze the internal geomagnetic field and its secular variation on both global and regional scales. This is an ongoing process as the current predictive IGRF is replaced with more accurate DGRF models while new predictive models are adopted for the next 5-years. To illustrate the continuing need for update, the adopted IGRFs for epochs 1995.0 and 2000.0 were compared with the final adopted DGRFs for the 1995.0 and 2000.0 using a simple difference method. Global differences were computed and plots of them for $X, Y$, and $Z$ components are shown in Figs. 1 and 2. Note that the DGRF2000 as added in the 9th generation IGRF is a degree/order 13 model, but IGRF2000 as added in the 8th generation IGRF is a degree/order 10 model. In order to avoid plots showing a lot of degree 1013 features, the DGRF2000 model was considered up to de- 
gree/order 10. The differences observed for 1995.0 epochs are, generally speaking, one order of magnitude larger then those for 2000.0.

For the 1995.0 epoch some very large differences (hundreds of nT) occur, predominantly in the Southern Hemisphere and in the $Z$ component. This is mainly due to the uneven distribution and reduced number of available data used in developing the 8th generation IGRF. The Ørsted satellite brought significant improvements in data coverage, and in the new IGRF models. As a result of the improved data quality and coverage, higher degree and order spherical harmonics are now feasible. We believe that the new modelling techniques and the forthcoming missions, such as Swarm (Friis-Christensen et al., 2004) will dramatically improve our field knowledge, as well as the IGRF/DGRF models.

Acknowledgments. My personal thanks for the support received from various organizations and individuals in the international efforts to produced IGRF/DGRF models. I would like to thank Susan McLean, Tatiana Bondar and the guest editor Susan Macmillan for useful suggestions that helped in improving the paper. All maps were plotted using the GMT software (Wessel and Smith, 1998). This is IPGP contribution 2055.

\section{References}

Cain, J. C., S. J. Hendricks, R. A. Langel, and W. V. Hudson, A proposed model for the International Geomagnetic Reference Field 1965, J. Geomag. Geoelectr., 19, 335-355, 1967.
Chambodut, A., J. Schwarte, B. Langlais, H. Lühr, and M. Mandea, The selection of data in field modeling, Proceedings 4th OIST ( $\emptyset$ rsted International Science Team conference) meeting, 31-34, 2003.

Chambodut, A., B. Langlais, and M. Mandea, Candidate main-field models for the Definitive Geomagnetic Reference Field 1995.0 and 2000.0, Earth Planets Space, 57, this issue, 1197-1202, 2005.

Friis-Christensen, E., A. De Santis, A. Jackson, G. Hulot, A. Kuvshinov, H. Lühr, M. Mandea, S. Maus, N. Olsen, M. Purucker, M. Rothacher, T. Sabaka, A. Thomson, S. Vennerstrom, and P. Visser, Swarm. The Earth's Magnetic Field and Environment Explorers, ESA SP-1279(6), 2004.

Holme, R., Modelling of attitude error in vector magnetic data: application to Oersted data, Earth Planets Space, 52, 1189-1197, 2000.

Langlais, B. and M. Mandea, An IGRF candidate main geomagnetic field model for epoch 2000 and a secular variation model for 2000-2005, Earth Planets Space, 52, 1137-1148, 2000.

Olsen, N., A model of the geomagnetic field and its secular variation for epoch 2000, Geophys. J. Int., 149, 454-462, 2002.

Olsen, N., F. Lowes, and T. J. Sabaka, Ionospheric and induced field leakage in geomagnetic field models, and derivation of candidate models for DGRF 1995 and DGRF 2000, Earth Planets Space, 57, this issue, 1191-1196, 2005.

Sabaka, T., N. Olsen, and R. A. Langel, A comprehensive model of the quiet-time, near-Earth magnetic field: phase 3, Geophys. J. Int., 151, 32-68, 2002.

Sabaka, T., N. Olsen, and M. E. Purucker, Extending comprehensive models of the Earth's magnetic field with Ørsted and CHAMP data, Geophys. J. Int., 159, 521-547, 2004.

Wessel, P. and W. H. F. Smith, New, improved version of the Generic Mapping Tools released, EOS Trans. AGU, 79, 579, 1998.

M. Mandea (e-mail: mioara@gfz-potsdam.de) 